\title{
Fucose-Migration in intakten protonierten Glykan-Ionen - ein univer- selles Phänomen in der Massenspektrometrie
}

\author{
Eike Mucha, Maike Lettow, Mateusz Marianski, Daniel A. Thomas, Weston B. Struwe, \\ David J. Harvey, Gerard Meijer, Peter H. Seeberger, Gert von Helden* und Kevin Pagel*
}

\begin{abstract}
Fucose ist ein essenzieller Desoxyzucker, der in einer Vielzahl biologisch relevanter Glykane und ihrer Konjugate vorkommt. Ein verbreitetes Problem in massenspektrometrischen Analysen von fucosylierten Glykanen ist der intramolekulare Transfer von Fucoseeinheiten, der zu fehlerhaften Sequenzzuordnungen führen kann. Diese Migrationsreaktion wird typischerweise der Aktivierung während der kollisionsinduzierten Dissoziation in der Tandem-Massenspektrometrie (Tandem-MS) zugeordnet. Hier wird erstmals mit KaltionenSpektroskopie gezeigt, dass Fucose-Migration nicht auf Fragmente beschränkt ist, die in der Tandem-MS erhalten werden, sondern auch in intakten Glykan-Ionen beobachtet werden kann. Diese Beobachtung lässt auf eine mögliche niedrige Energiebarriere für diese Transferreaktion schließen und verallgemeinert die Fucose-Migration zu einem Problem, das bei jeder Art von Massenspektrometrie-Experiment auftreten kann.
\end{abstract}

Kohlenhydrate - oder Glykane - sind eine der vier wichtigsten Klassen von Biomolekülen. Ihre immense Strukturvielfalt ermöglicht vielfältige biologische Funktionen wie Zellsignalisierung oder molekulare Erkennung. ${ }^{[1]}$ Die komplexe Natur von Glykanen erschwert jedoch detaillierte

[*] E. Mucha, M. Lettow, Dr. M. Marianski, Dr. D. A. Thomas, Prof. Dr. G. Meijer, Prof. Dr. G. von Helden, Prof. Dr. K. Pagel

Fritz-Haber-Institut der Max-Planck-Gesellschaft

Faradayweg 4-6, 14195 Berlin (Deutschland)

E-Mail: helden@fhi-berlin.mpg.de

E. Mucha, M. Lettow, Prof. Dr. K. Pagel

Institut für Chemie und Biochemie

der Freien Universität Berlin

Takustraße 3, 14195 Berlin (Deutschland)

E-Mail: kevin.pagel@fu-berlin.de

Prof. Dr. P. H. Seeberger

Max-Planck-Institut für Kolloid- und Grenzflächenforschung

Potsdam (Deutschland)

und

Institut für Chemie und Biochemie

der Freien Universität Berlin (Deutschland)

Dr. W. B. Struwe

Oxford Glycobiology Institute

Department of Biochemistry

University of Oxford (Großbritannien)

Dr. D. J. Harvey

Target Discovery Institute

Nuffield Department of Medicine

University of Oxford (Großbritannien)

Hintergrundinformationen und die Identifikationsnummer (ORCID)

eines Autors sind unter:

https://doi.org/10.1002/ange.201801418 zu finden.
Strukturanalysen. Typischerweise werden anspruchsvolle Massenspektrometrie(MS)-basierte Techniken in Kombination mit Chromatographie ${ }^{[2]}$ oder Ionenmobilitäts-Spektrometrie $^{[3]}$ eingesetzt. Eine weitere leistungsfähige Methode zur Untersuchung von Molekülen in der Gasphase ist die Kombination aus MS und Infrarot(IR)-Spektroskopie. Dieser Ansatz wird beispielsweise für Peptide und Proteine genutzt, um Informationen über funktionelle Gruppen, Sekundärstrukturelemente oder bevorzugte Molekülkonformationen abzuleiten. Neuere Studien zeigen, dass auch für Kohlenhydrate hochdiagnostische IR-Fingerabdrücke durch Kaltionen-Spektroskopie erhalten werden können. ${ }^{[4]}$ Dies gilt nicht nur für intakte Glykan-Ionen, sondern auch für deren diagnostische Fragmente, die oft zur Strukturaufklärung genutzt werden. ${ }^{[5]}$

Kürzlich untersuchten wir isomere, fucosehaltige Tetrasaccharide mit Kaltionen-IR-Spektroskopie und zeigten ihre charakteristischen, optischen Signaturen, die eine eindeutige Unterscheidung ermöglichen. ${ }^{[4]}$ Fucose ist ein weit verbreiteter Monosaccharid-Baustein, der in $\mathrm{N}$-Glykanen, $\mathrm{O}$-Glykanen oder Milchzuckern enthalten ist und typischerweise im letzten Schritt der Glykan-Prozessierung eingebaut wird. Im Unterschied zu den meisten Monosacchariden fehlt Fucose die Hydroxygruppe an der C-6-Position, weshalb sie ein so genannter Desoxyzucker ist (Abbildung 1b). Biologisch relevante Beispiele fucosehaltiger Glykane sind das ABO- und Lewis-Blutgruppensystem. Darunter befindet sich auch das Lewis-b(Le $\left.{ }^{b}\right)$-Antigen, ein fucosyliertes TetrasaccharidEpitop, das im menschlichen Körper weit verbreitet ist. Das isomere Lewis-y $\left(\mathrm{Le}^{\mathrm{y}}\right)$-Antigen hingegen wird auf einer Vielzahl von Tumoren exprimiert und kann als Biomarker genutzt werden $^{[6]}$ (Abbildung 1c). Glykokonjugate wie Glykoproteine weisen häufig charakteristische Fucosylierungsmuster auf,
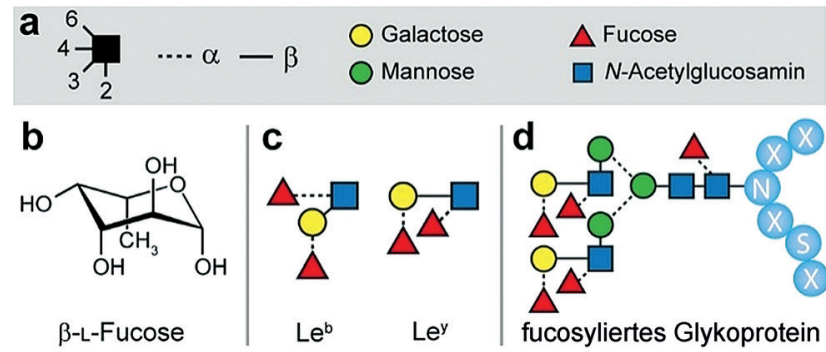

Abbildung 1. a) Symbolnomenklatur für Glykane $\left(\mathrm{SNFG}^{[8]}\right)$. b) L-Fucose ist ein ubiquitärer Monosaccharidbaustein, dem eine Hydroxygruppe an der C-6-Position fehlt. c) Lewis-b- und Lewis-y-Tetrasaccharide als Beispiele für fucosylierte Oligosaccharide. d) Ein stark fucosyliertes $N$ Glykan, das aus der Ohrspeicheldrüse extrahiert wurde. ${ }^{[9]}$ 
die sich bei Krebserkrankungen dynamisch verändern können. ${ }^{[7]}$

Ein häufig beobachtetes Phänomen für fucosehaltige Glykane in Tandem-MS-Experimenten ist der intramolekulare Transfer von terminalen Fucoseeinheiten zu benachbarten oder entfernten Monosacchariden. ${ }^{[10]}$ Diese so genannte Fucose-Migration führt oft zu irreführenden Fragmenten, aus denen wiederum fehlerhafte Strukturzuordnungen resultieren können. Hier zeigen wir eine spektroskopische Untersuchung von fucosylierten Glykanen und präsentieren den ersten direkten Beleg für Fucose-Migration in intakten protonierten Glykan-Ionen. Unsere Ergebnisse zeigen, dass Fucose-Migration nicht nur in Tandem-MS-Experimenten vorkommt, sondern ein allgemeines Problem in allen Bereichen der Massenspektrometrie ist.

Der experimentelle Aufbau wurde zuvor ausführlich beschrieben, und wir verweisen die interessierten Leser auf die entsprechende Literatur. ${ }^{[4 a, 11]}$ Kurz gefasst werden molekulare Ionen durch Nanoelektrosprayionisation erzeugt, nach ihrem Masse-zu-Ladungs-Verhältnis mittels QuadrupolMassenfilter ausgewählt und in einer Hexapol-Ionenfalle gesammelt. Suprafluide Heliumtröpfchen mit durchschnittlich $10^{5}$ Heliumatomen durchqueren anschließend die Falle und nehmen Ionen auf, die danach schnell auf die Gleichgewichtstemperatur des Tröpfchens $(0.4 \mathrm{~K})$ abgekühlt werden. Daraufhin werden die nun kalten Ionen in den Heliumtröpfchen mit dem IR-Freie-Elektronen-Laser des FritzHaber-Instituts (FHI-FEL ${ }^{[12]}$ ) untersucht, und es wird ein hoch reproduzierbares IR-Spektrum aufgenommen. Für intakte Glykan-Ionen werden sanfte Transferbedingungen verwendet, um eine übermäßige Aktivierung der Ionen beim Übergang in das Massenspektrometer zu vermeiden (Abbildung S1 der Hintergrundinformationen). Fragmente hingegen werden durch harschere Bedingungen erhalten, bei denen die Dissoziation von intakten Glykan-Ionen induziert wird (Abbildung S2).

Zunächst wurden Glykane der Le ${ }^{\mathrm{y}}$-Reihe als Natriumaddukte untersucht. Die Aktivierung der $\left[\mathrm{Le}^{\mathrm{y}}+\mathrm{Na}\right]^{+}$-Vorläuferionen $(m / z=698)$ erzeugt ein intensives Signal, das aus dem neutralen Verlust einer einzelnen Fucoseeinheit hervorgeht $(m / z=552)$. Je nachdem, welcher der beiden Fucosereste bei der Fragmentierung verloren geht, sind zwei verschiedene Fragmentstrukturen möglich (Abbildung 2a). Da diese beiden Trisaccharidfragmente die gleiche Masse aufweisen, kann eine massenspektrometrische Analyse allein nicht zeigen, ob eines der beiden Fragmente vorzugsweise gebildet wird. Beide Trisaccharidstrukturen sind jedoch auch als Standards verfügbar: Lewis $\mathrm{x}\left(\mathrm{Le}^{\mathrm{x}}\right)$ und das Blutgruppenantigen $\mathrm{H}-2$ (BG-H2). Unter Verwendung des oben beschriebenen experimentellen Aufbaus wurden IR-Spektren für die Natriumaddukte des $\mathrm{Le}^{\mathrm{y}}$-Fragments sowie der Trisaccharidstandards $\mathrm{Le}^{\mathrm{x}}$ und $\mathrm{BG}-\mathrm{H} 2$ aufgezeichnet (Abbildung $2 b$ ). Alle drei IR-Spektren zeigen gut aufgelöste Absorptionsmerkmale zwischen $\tilde{v}=1000$ und $1700 \mathrm{~cm}^{-1}$. Starke Absorptionen um $\tilde{v}=1100 \mathrm{~cm}^{-1}$ werden dabei üblicherweise CO-Streckschwingungen zugeschrieben. Die Banden bei $\tilde{v}=$ 1520 und $1680 \mathrm{~cm}^{-1}$ hingegen stammen wahrscheinlich aus den charakteristischen Bereichen Amid II (NH-Biegeschwingungen) und Amid I ( $\mathrm{C}=\mathrm{O}$-Streckschwingungen $)$ des
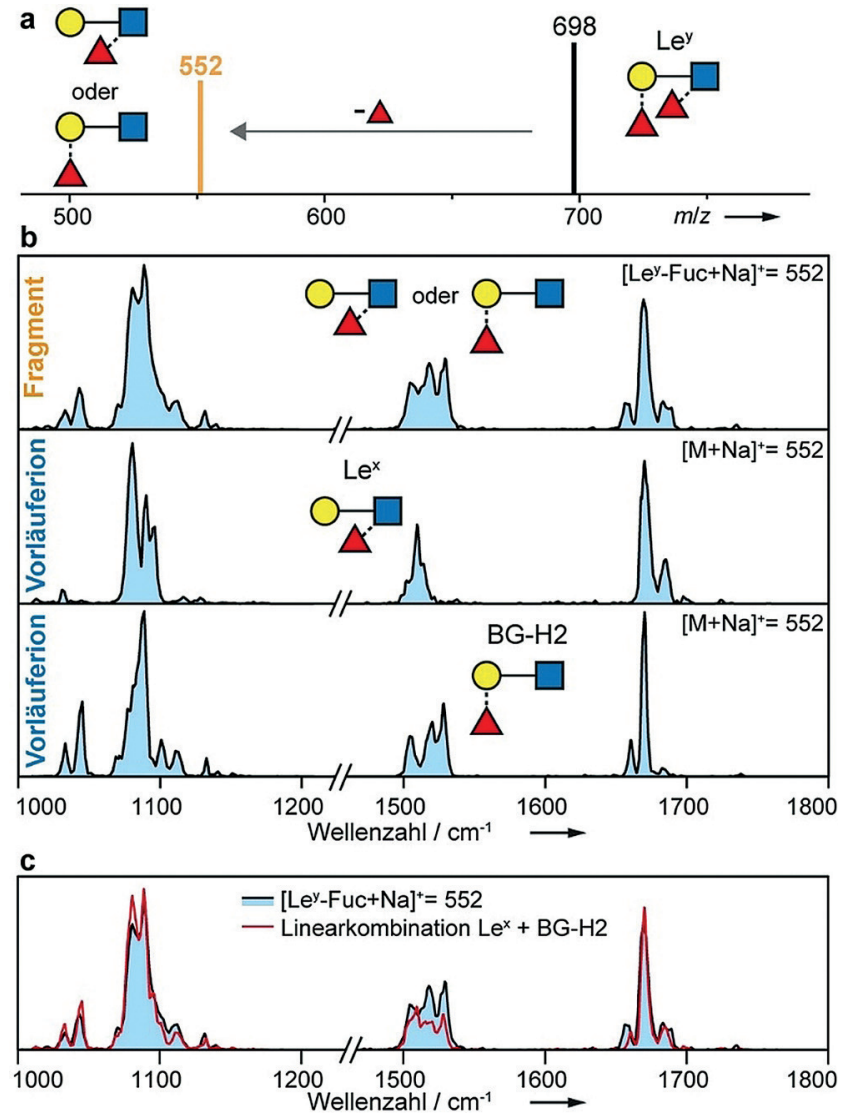

Abbildung 2. a) Schematisches Tandem-MS-Spektrum des Le ${ }^{y}$-Tetrasac charids als $[M+N a]^{+}$-Spezies. Das Hauptfragment $(m / z=552)$ resultiert aus dem neutralen Verlust einer Fucoseeinheit. Das experimentelle Massenspektrum ist in den Hintergrundinformationen gezeigt. b) IR-Spektren des $\left[\mathrm{Le}^{\mathrm{y}} \text {-Fuc }+\mathrm{Na}\right]^{+}$-Fragments und der intakten Standards Le ${ }^{x}$ und BG-H2 $(m / z=552)$. c) Das IR-Spektrum des Le'-Fragments als Natriumaddukt kann durch eine Linearkombination der individuellen Trisaccharidspektren von $\mathrm{Le}^{\mathrm{x}}$ und $\mathrm{BG}-\mathrm{H} 2$ dargestellt werden.

$N$-Acetylglucosaminrestes. Während die beiden Trisaccharide $\mathrm{Le}^{\mathrm{x}}$ und BG-H2 eindeutig durch ihre einzigartigen spektralen Signaturen unterschieden werden können, weist das $\mathrm{Le}^{\mathrm{y}}$ Fragmentspektrum Absorptionsmerkmale der beiden Trisaccharidspektren auf. Wenn beide Trisaccharide während der Fragmentierung gebildet werden, kann man davon ausgehen, dass das resultierende IR-Spektrum eine Linearkombination der beiden individuellen Trisaccharidspektren ist. Tatsächlich zeigt die Linearkombination (Abbildung 2c) eine gute Übereinstimmung mit dem Fragmentspektrum und lässt auf ein ungefähres Fragmentverhältnis von 1:1 schließen (Lex/BG-H2).

Frühere Studien verwendeten Ionenmobilitäts-Massenspektrometrie (IM-MS), um Lewis- und Blutgruppenantigene sowie ihre charakteristischen Fragment-Ionen zu untersuchen. Für die Natriumaddukte der Le ${ }^{\mathrm{x}}$ - und BG-H2-Trisaccharide wurden charakteristische Kollisionsquerschnitte (collision cross-section, CCS) erhalten, und beide Strukturen konnten nach Aktivierung des $\mathrm{Le}^{\mathrm{y}}$-Vorläuferions als Fragmente identifiziert werden. ${ }^{[3 b]}$ Die protonierten Spezies zeigen dagegen sowohl für die Trisaccharide als auch für das Le $^{\mathrm{y}}$-Fragment identische CCS-Werte (siehe Hintergrundin- 
formationen). Um zu untersuchen, inwieweit ähnliche CCSWerte für diese protonierten Glykane auf ähnliche dreidimensionale Strukturen zurückzuführen sind, wurde die Kaltionen-IR-Spektroskopie eingesetzt.

Die protonierten $\mathrm{Le}^{\mathrm{y}}$-Vorläuferionen $(\mathrm{m} / \mathrm{z}=676)$ weisen ein ähnliches Fragmentierungsmuster wie die zuvor beschriebenen Natriumaddukte auf. Ein starkes Signal entspricht dem neutralen Verlust einer einzelnen Fucoseeinheit $(\mathrm{m} / z=530)$. Abhängig davon, welche Fucoseeinheit bei der Fragmentierung verloren geht, sind erneut zwei resultierende Fragmentstrukturen möglich (Abbildung 3a). Das entspre-
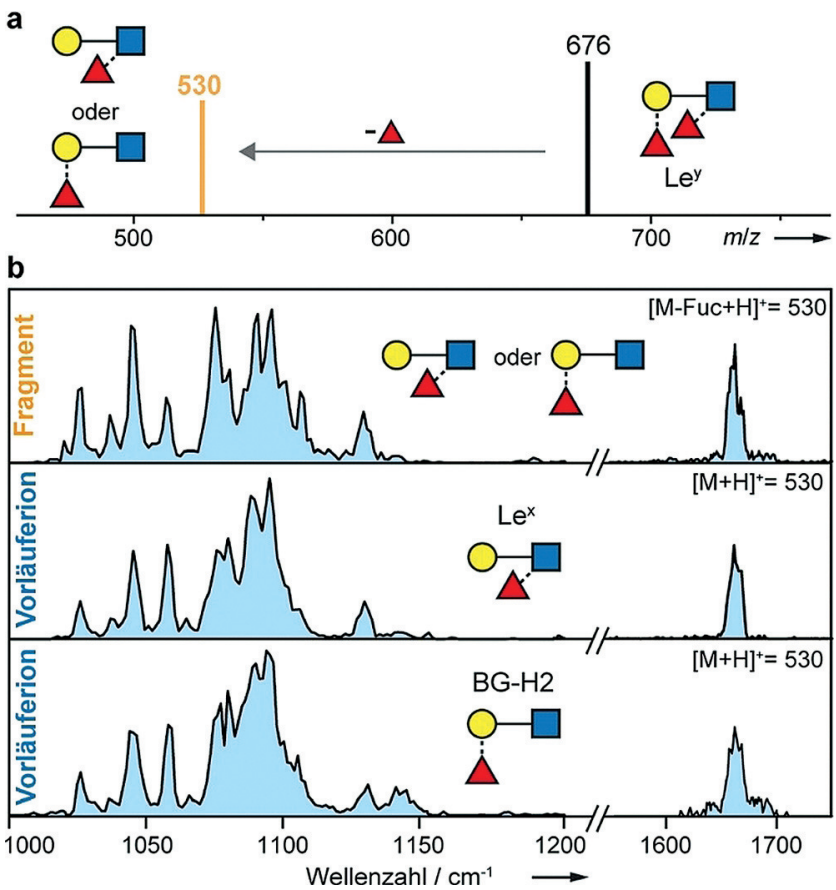

Abbildung 3. a) Schematisches Tandem-MS-Spektrum des Le'-Tetrasaccharids als $[M+H]^{+}$-Spezies. Das Hauptfragment $(m / z=530)$ resultiert aus dem neutralen Verlust einer Fucoseeinheit. Das experimentelle Massenspektrum ist in den Hintergrundinformationen gezeigt. b) IRSpektren des $\left[\mathrm{Le}^{\mathrm{y}} \text {-Fuc }+\mathrm{H}\right]^{+}$-Fragments sowie der intakten Standards $\mathrm{Le}^{\mathrm{x}}$ und BG-H2 (m/z 530).

chende IR-Spektrum dieses Fragments wurde aufgezeichnet (Abbildung 3b) und zeigt eine Vielzahl von aufgelösten Banden zwischen $\tilde{v}=1000$ und $1200 \mathrm{~cm}^{-1}$ und eine einzelne Bande um $\tilde{v}=1680 \mathrm{~cm}^{-1}$. Als nächstes wurden die beiden Trisaccharid-Standards $\mathrm{Le}^{\mathrm{x}}$ und $\mathrm{BG}-\mathrm{H} 2$ einzeln untersucht, da sie die beiden verschiedenen Strukturen der möglichen Fragment-Ionen aufweisen. Interessanterweise sind die IRSpektren der protonierten Vorläuferionen $\mathrm{Le}^{\mathrm{x}}$ und BG-H2 sowie das Le ${ }^{\mathrm{y}}$-Fragmentspektrum nahezu identisch. Jede signifikante Absorptionsbande ist in den einzelnen Spektren vorhanden, und nur die relative Intensität einiger Banden unterscheidet sich leicht zwischen dem Le ${ }^{\mathrm{y}}$-Fragment und den Trisaccharid-Standards. Dieser Befund ist besonders überraschend und im Widerspruch zu früheren Ergebnissen, die zeigen, dass diese experimentelle Technik in der Lage ist, selbst kleinste Strukturvariationen, wie die stereochemische Orientierung einer einzelnen Hydroxygruppe, aufzulösen.
Doch warum liefert diese hochdiagnostische Methode identische IR-Signaturen für diese Strukturisomere?

Die Wahrscheinlichkeit, dass beide Trisaccharide zufällig das gleiche IR-Spektrum ergeben, ist vernachlässigbar gering, sodass nur eine einzige plausible Erklärung bleibt: Sowohl das protonierte $\mathrm{Le}^{\mathrm{y}}$-Fragment als auch die beiden protonierten Trisaccharidstandards $\mathrm{Le}^{\mathrm{x}}$ und BG-H2 durchlaufen eine Umlagerungsreaktion - eine Fucose-Migration - und konvergieren zur selben Struktur, die wiederum zu den drei identischen IR-Signaturen führt. Eine mögliche Erklärung für leicht unterschiedliche Absorptionsintensitäten einzelner Banden (wie die Bande bei $\tilde{v}=1040 \mathrm{~cm}^{-1}$ ) ist ein anderes Verhältnis von $\alpha$ - und $\beta$-Anomeren zwischen den intakten Ionen und dem aktivierten Fragment. Die schwache Absorptionsbande bei $\tilde{v}=1145 \mathrm{~cm}^{-1}$, die nur im Spektrum von BG-H2 gefunden wird, stammt möglicherweise von einem kleinen Bruchteil einer anderen Molekülkonformation oder eines anderen Umlagerungsprodukts.

Im Unterschied zu früheren Tandem-MS-Experimenten, die indirekt eine Fucose-Migration durch das Auftreten irreführender Fragmente nachweisen, sind diese Ergebnisse der erste direkte Beleg für diese rätselhafte Umlagerungsreaktion. In der Literatur $^{[10 a]}$ wurde vorgeschlagen, dass die Fucose-Migration durch Aktivierung der Ionen während der kollisionsinduzierten Dissoziation (CID) von Vorläuferionen verursacht wird. Der Nachweis dieser Umlagerung in intakten Ionen zeigt jedoch deutlich, dass Fragmentierung keine Voraussetzung für die Fucose-Migration ist. Die Energiebarriere für diese Reaktion kann wahrscheinlich nicht verallgemeinert werden und hängt stark von der Glykanstruktur ab. Hier wurden jedoch sanfte Transferbedingungen verwendet, um die intakten Trisaccharid-Ionen zu untersuchen und die Aktivierung während des Übergangs in das Massenspektrometer zu minimieren. Daher kann für diesen Prozess eine niedrige Aktivierungsenergie erwartet werden. Die Natriumaddukte durchlaufen hingegen keine Fucose-Migration und zeigen daher charakteristische IR-Signaturen (Abbildung 2 b). Diese Beobachtung stimmt mit früheren Studien überein, die ergaben, dass die Fucose-Migration unter Verwendung von Natrium- anstelle von Protonenaddukten effizient unterdrückt werden kann.

Transferreaktionen von Fucoseeinheiten sind im Allgemeinen mit dem Vorhandensein mobiler Protonen assoziiert, aber das endgültige Strukturmotiv sowie der zugrunde liegende Mechanismus sind noch nicht aufgeklärt. Um Einblicke in den Reaktionsmechanismus der Fucose-Migration zu erhalten, können die hochaufgelösten IR-Spektren dieser Arbeit prinzipiell mit denen von Strukturkandidaten verglichen werden, die aus Ab-initio-Methoden generiert werden. Allerdings machen die große Anzahl von möglichen Umlagerungsprodukten und der unbekannte Mechanismus dies zu einer äußerst schwierigen, wenn nicht praktisch unmöglichen Aufgabe.

Interessanterweise zeigen die verwandten isomeren Trisaccharide Lewis a (Le ${ }^{a}$ ) und BG-H Typ 1 (BG-H1) der Lewis-b-Reihe sowohl für die protonierten Spezies als auch für die Natriumaddukte unterschiedliche IR-Signaturen (Abbildungen S3 und S4). Die unterschiedlichen IR-Spektren der protonierten $\mathrm{Le}^{\mathrm{a}}$ - und BG-H1-Trisaccharide haben eine 
gewisse Ähnlichkeit mit dem einfach defucosylierten Lewisb-Fragment. Diese Beobachtung kann darauf hinweisen, dass die Fucose-Migration für diese Strukturen entweder inhibiert ist oder dass die Produkte nach einer möglichen Umlagerungsreaktion unterschiedlich sind.

Die Kaltionen-Infrarot-Spektroskopie zeigt, dass FucoseMigration in intakten Ionen auftreten kann und nicht notwendigerweise ein Ergebnis der kollisionsinduzierten Dissoziation (CID) ist. Daher muss die Fucose-Migration als ein allgemeines Problem in der massenspektrometrischen Analyse von protonierten Glykan-Ionen betrachtet werden, das universell bei jeder Art von MS-Experiment auftreten kann. Der genaue Mechanismus und das Endprodukt der Transferreaktion sind noch nicht bekannt, aber es ist wahrscheinlich, dass mobile Protonen die Umlagerung einzelner Fucosereste zu räumlich benachbarten Stellen katalysieren. Das Beobachten von Fucose-Migrationsreaktionen in intakten, protonierten Vorläuferionen lässt weiterhin auf eine möglicherweise niedrige Aktivierungsenergie schließen. In Metalladdukten kann hingegen kein Protonentransfer auftreten, und die lokalisierte Ladung verhindert die Umlagerungsreaktion. Weitere Experimente unter Verwendung von spezifisch isotopenmarkierten Glykanen und umfangreiche Rechnungen werden erforderlich sein, um die zugrunde liegenden Prozesse vollständig aufzuklären.

\section{Danksagung}

Wir danken der Max-Planck-Gesellschaft für finanzielle Förderung sowie Sandy Gewinner und Dr. Wieland Schöllkopf für ihre kenntnisreiche Unterstützung beim Einsatz des Freie-Elektronen-Lasers am FHI. Außerdem danken wir Dr. Fabian Pfrengle für hilfreiche Diskussionen. DAT ist dankbar für die Unterstützung durch die Alexander von HumboldtStiftung.

\section{Interessenkonflikt}

Die Autoren erklären, dass keine Interessenkonflikte vorliegen.

Stichwörter: Fucose-Migration - Glykane - IR-Spektroskopie · Kohlenhydrate Massenspektrometrie

Zitierweise: Angew. Chem. Int. Ed. 2018, 57, 7440-7443 Angew. Chem. 2018, 130, 7562-7565

[1] a) A. Varki, Glycobiology 1993, 3, 97-130; b) R. A. Dwek, Chem. Rev. 1996, 96, 683-720.

[2] a) K. Mariño, J. Bones, J. J. Kattla, P. M. Rudd, Nat. Chem. Biol. 2010, 6, 713; b) L. Veillon, Y. Huang, W. Peng, X. Dong, B. G. Cho, Y. Mechref, Electrophoresis 2017, 38, 2100-2114; c) N.
Viseux, E. de Hoffmann, B. Domon, Anal. Chem. 1997, 69, 3193 -3198; d) L. R. Ruhaak, A. M. Deelder, M. Wuhrer, Anal. Bioanal. Chem. 2009, 394, 163-174.

[3] a) J. Hofmann, H. S. Hahm, P. H. Seeberger, K. Pagel, Nature 2015, 526, 241-244; b) J. Hofmann, A. Stuckmann, M. Crispin, D. J. Harvey, K. Pagel, W. B. Struwe, Anal. Chem. 2017, 89, $2318-2325$; c) P. Both, A. P. Green, C. J. Gray, R. Sardzik, J. Voglmeir, C. Fontana, M. Austeri, M. Rejzek, D. Richardson, R. A. Field, G. Widmalm, S. L. Flitsch, C. E. Eyers, Nat. Chem. 2014, 6, 65-74; d) H. Hinneburg, J. Hofmann, W. B. Struwe, A. Thader, F. Altmann, D. Varon Silva, P. H. Seeberger, K. Pagel, D. Kolarich, Chem. Commun. 2016, 52, $4381-4384$.

[4] a) E. Mucha, A. I. González Flórez, M. Marianski, D. A. Thomas, W. Hoffmann, W. B. Struwe, H. S. Hahm, S. Gewinner, W. Schollkopf, P. H. Seeberger, G. von Helden, K. Pagel, Angew. Chem. Int. Ed. 2017, 56, 11248-11251; Angew. Chem. 2017, 129, $11400-11404$; b) C. Masellis, N. Khanal, M. Z. Kamrath, D. E. Clemmer, T. R. Rizzo, J. Am. Soc. Mass Spectrom. 2017, 28, $2217-2222$.

[5] B. Schindler, L. Barnes, G. Renois, C. Gray, S. Chambert, S. Fort, S. Flitsch, C. Loison, A.-R. Allouche, I. Compagnon, Nat. Commun. 2017, 8, 973.

[6] a) B. W. T. Yin, C. L. Finstad, K. Kitamura, M. G. Federici, M. Welshinger, V. Kudryashov, W. J. Hoskins, S. Welt, K. O. Lloyd, Int. J. Cancer 1996, 65, 406-412; b) X. Xie, M. Boysen, O. P. F. Clausen, M. A. Bryne, Laryngoscope 1999, 109, 1474-1480; c) R. B. Myers, S. Srivastava, W. E. Grizzle, J. Urol. 1995, 153, $1572-1574$

[7] J. Le Pendu, S. Marionneau, A. Cailleau-Thomas, J. Rocher, B. Le Moullac-Vaidye, M. Clément, APMIS 2001, 109, 9-26.

[8] A. Varki, R. D. Cummings, M. Aebi, N. H. Packer, P. H. Seeberger, J. D. Esko, P. Stanley, G. Hart, A. Darvill, T. Kinoshita, J. J. Prestegard, R. L. Schnaar, H. H. Freeze, J. D. Marth, C. R. Bertozzi, M. E. Etzler, M. Frank, J. F. Vliegenthart, T. Lutteke, S. Perez, E. Bolton, P. Rudd, J. Paulson, M. Kanehisa, P. Toukach, K. F. Aoki-Kinoshita, A. Dell, H. Narimatsu, W. York, N. Taniguchi, S. Kornfeld, Glycobiology 2015, 25, 1323-1324.

[9] G. R. Guile, D. J. Harvey, N. O'Donnell, A. K. Powell, A. P. Hunter, S. Zamze, D. L. Fernandes, R. A. Dwek, D. R. Wing, Eur. J. Biochem. 1998, 258, 623-656.

[10] a) D. J. Harvey, T. S. Mattu, M. R. Wormald, L. Royle, R. A. Dwek, P. M. Rudd, Anal. Chem. 2002, 74, 734-740; b) A. H. Franz, C. B. Lebrilla, J. Am. Soc. Mass Spectrom. 2002, 13, $325-$ 337; c) M. Wuhrer, C. A. M. Koeleman, C. H. Hokke, A. M. Deelder, Rapid Commun. Mass Spectrom. 2006, 20,1747-1754; d) C. Nwosu, H. K. Yau, S. Becht, Anal. Chem. 2015, 87, 59055913.

[11] a) F. Filsinger, D. S. Ahn, G. Meijer, G. von Helden, Phys. Chem. Chem. Phys. 2012, 14, 13370-13377; b) A. I. González Flórez, E. Mucha, D. S. Ahn, S. Gewinner, W. Schöllkopf, K. Pagel, G. von Helden, Angew. Chem. Int. Ed. 2016, 55, 3295-3299; Angew. Chem. 2016, 128, 3356-3360.

[12] W. Schöllkopf, S. Gewinner, H. Junkes, A. Paarmann, G. von Helden, H. Bluem, A. M. M. Todd, Proc. SPIE-Int. Soc. Opt. Eng. 2015, 9512, 95121L.

Manuskript erhalten: 2. Februar 2018

Veränderte Fassung erhalten: 23. März 2018

Akzeptierte Fassung online: 24. April 2018

Endgültige Fassung online: 25. Mai 2018 